\title{
Tumor Cells and Cancer-Associated Fibroblasts: A Synergistic Crosstalk to Promote Thyroid Cancer
}

\author{
Laura Fozzatti ${ }^{1}$, Sheue-yann Cheng ${ }^{2}$ \\ ${ }^{1}$ Center for Research in Clinical Biochemistry and Immunology (CIBICI)-National Scientific and Technical Research Council \\ (CONICET), School of Chemical Sciences, National University of Córdoba, Cordoba, Argentina; ${ }^{2}$ Laboratory of Molecular \\ Biology, Center for Cancer Research, National Cancer Institute, National Institutes of Health, Bethesda, MD, USA
}

Thyroid cancer is the most common endocrine malignancy. Although most thyroid cancer patients are successfully treated and have an excellent prognosis, a percentage of these patients will develop aggressive disease and, eventually, progress to anaplastic thyroid cancer. Since most patients with this type of aggressive thyroid carcinoma will die from the disease, new treatment strategies are urgently needed. Tumor cells live in a complex and dynamic tumor microenvironment composed of different types of stromal cells. Cancer-associated fibroblasts (CAFs) are one of the most important cell components in the tumor microenvironment of most solid tumors, including thyroid cancer. CAFs originate mainly from mesenchymal cells and resident fibroblasts that are activated and reprogrammed in response to paracrine factors and cytokines produced and released by tumor cells. Upon reprogramming, which is distinguished by the expression of different marker proteins, CAFs synthesize and secret soluble factors. The secretome of CAFs directly impacts different functions of tumor cells. This bi-directional interplay between CAFs and tumor cells within the tumor microenvironment ends up fostering tumor cancer progression. CAFs are therefore key regulators of tumor progression and represent an under-explored therapeutic target in thyroid cancer.

Keywords: Thyroid carcinoma; Tumor microenvironment; Cancer-associated fibroblasts; Paracrine communication

\section{INTRODUCTION}

Thyroid cancer is relatively rare, representing approximately $3 \%$ of all new cancer cases in the United States [1]. It is, however, the most common cancer in the endocrine system, and it has had the fastest growing incidence of all cancers over the last decades [2]. Among malignant tumors affecting women, its incidence ranks fifth worldwide [3]. In the United States, about 52,900 new cases of thyroid cancer are expected to be diagnosed in 2020 [1].
Received: 2 September 2020, Revised: 16 October 2020,

Accepted: 20 October 2020

Corresponding authors: Laura Fozzatti

Center for Research in Clinical Biochemistry and Immunology (CIBICI)-National Scientific and Technical Research Council (CONICET), Haya de la Torre y Medina Allende, X5000HUA, Cordoba, Argentina

Tel: +54-351-535-3851, Fax: +54-351-433-3048, E-mail: 1fozzatti@fcq.unc.edu.ar

\section{Copyright $(\odot 2020$ Korean Endocrine Society}

This is an Open Access article distributed under the terms of the Creative Commons Attribution Non-Commercial License (https://creativecommons.org/ licenses/by-nc/4.0/) which permits unrestricted non-commercial use, distribution, and reproduction in any medium, provided the original work is properly cited.

Sheue-yann Cheng

Laboratory of Molecular Biology, Center for Cancer Research, National Cancer

Institute, National Institutes of Health. 37 Convent Drive, Building 37, Room

5128, Bethesda, MD 20892-4264, USA

Tel: +1-240-760-7828, Fax: +1-240-541-4498, E-mail: chengs@mail.nih.gov 
While alterations on some genes and pathways have been described to be associated with the different forms of thyroid cancer $[4,5]$, the most recent evidence indicates that the development and progression of cancer depend not only on genetic characteristics but also on the interaction of tumor cells and their surrounding microenvironment $[6,7]$. This tumor microenvironment (TME) is an active and dynamic component influencing cancer cell behavior, disease progression, and response to therapy in many different types of neoplasia. The TME comprises a variety of non-epithelial cells (or stromal cells). Fibroblasts are one of the main cell components of the TME [8-11].

Although fibroblasts were first described at the end of the nineteenth century [12], their role in tumor development has only recently begun to be uncovered [6]. Normal fibroblasts play an important part in tissue homeostasis and wound repair by secreting extracellular matrix (ECM) components. In non-tumor tissues they are generally quiescent. However, upon tissue damage, fibroblasts are activated to proliferate and increase their synthetic and metabolic activity. Activated fibroblasts secrete higher levels of ECM components to favor wound closure and scar formation $[13,14]$. Once the wound is repaired, most of these activated fibroblasts undergo apoptosis and their number decreases $[15,16]$. In contrast, tumors have been considered "wounds that do not heal" because the wound healing response is not self-limited and the number of activated fibroblasts is not decreased, as in normal wound repair [17]. Instead, fibroblasts in tumors, called cancer-associated fibroblasts (CAFs), are key players in tumor development [13].

Although studies analyzing the contribution of the components of the TME, particularly CAFs, in thyroid cancer progression have been limited, the thyroid TME has become an evolving field. In this review we summarize the current knowledge about the origin and markers of CAFs in thyroid cancer. We focus specifically on the recent progress in understanding how CAFs drive thyroid cancer development and progression, from the standpoint of tumor-stroma interactions. We will also discuss CAFs as a promising therapeutic target in thyroid cancer.

\section{THYROID CANCER}

\section{Epidemiology and molecular pathogenesis}

Thyroid carcinomas are classified as follicular-derived or Ccell-derived, according to their cell of origin. The majority of thyroid cancers originate from follicular cells, constituting approximately $95 \%$ of all cases. Follicular-derived thyroid malignancies are subdivided into differentiated thyroid cancer (DTC), poorly differentiated thyroid cancer (PDTC), and anaplastic thyroid cancer (ATC) [4,5]. DTC includes papillary thyroid cancer (PTC) and follicular thyroid cancer (FTC). PTC is the most frequent type of thyroid cancer and is found in more than $80 \%$ of cases. It tends to grow slowly and spreads first to local lymph nodes $[5,18]$. FTC is the second most frequent and represents $10 \%$ to $15 \%$ of thyroid cancer cases. As with PTC, FTC can grow into lymph nodes in the neck. However, FTC is more likely than PTC to grow into blood vessels and spread to distant areas, particularly lungs and bones [19,20]. Although PDTC (1\% to $15 \%)$ and ATC $(<1 \%)$ are uncommon tumors, they constitute a more aggressive follicular-derived thyroid carcinoma [21-23]. Parafollicular C cell-derived thyroid cancer, which constitutes only a small fraction of thyroid cancer (approximately $2 \%$ to $5 \%$ ), is classified as medullary thyroid cancer (MTC) and has distinct biological characteristics [24].

DNA sequencing studies have provided unique insights into the genetic basis for most thyroid cancers. The main oncogenic alterations of thyroid cancer occur in genes involved in mitogen-activated protein kinase (MAPK) and phosphatidylinositol 3 kinase (PI3K) signaling pathways, such as $B R A F, R A S$, and RET [25,26]. The $B R A F^{V 600 E}$ mutation is the most frequent genetic mutation in PTC with a prevalence of approximately $60 \%$ $[4,18]$. For FTC, point mutations in $R A S$ genes (mainly $N R A S$ and $H R A S$ ) are the most frequent, found in $40 \%$ to $50 \%$ of the cases [25]. Activating mutations in the RET proto-oncogene account for most cases of MTC [24]. Other genetic alterations are also found in thyroid cancer, including loss of tumor protein 53 (TP53) and activating mutations in telomerase reverse transcriptase (TERT) promoter in undifferentiated thyroid cancer [21, 23,27].

Most patients with DTC have good rates of long-term survival. Standard treatment involves surgery, radioactive iodine, and thyroid hormone therapy [28]. The 5-year survival rate is approximately $98 \%$ [1]. A small percentage of DTCs, however, may manifest aggressive behavior that cannot be controlled by conventional therapy. Moreover, ATC is one of the most aggressive and lethal human malignancies, with a short median time of survival (3 to 6 months). Standard therapy was not effective in some DTC and ATC patients. Therefore, there is an urgent need to identify new biological targets that can be translated into novel clinical approaches.

\section{THE TUMOR MICROENVIRONMENT}

The effects of oncogenic transformation of epithelial tumor cells 
on cancer progression have been well established. In 2000, Hanahan and Weinberg [29] proposed a list of six capabilities acquired by cancer cells during the process of malignant transformation. These six hallmarks of cancer consisted of uncontrolled proliferation, insensitivity to anti-growth signals, evasion of apoptosis, unlimited replicative potential, acquired capabilities in tissue invasion and metastasis, and sustained angiogenesis [29]. However, at that time, the heterogeneous and structurally complex nature of tumors was largely unexplored. More recently, the tumor hallmarks were updated to include TME factors that are required for malignant transformation [6,7]. Cancer cells themselves produce stimulatory factors that recruit and activate numerous normal stromal cell types from the neighboring tissues as well from the circulation. Interactions between neoplastic cancer cells and their supporting stroma create the TME, which profoundly shapes cancer progression [30]. As an example, pancreatic stellate cells (PSCs) have been reported to play a crucial role in the pathogenesis of pancreatic ductal adenocarcinoma (PDAC). PSCs represent the main cell type causing the desmoplastic reaction, which constitutes a dramatic increase in connective tissue or stromal cells that surround and infiltrate the tumor. The desmoplastic stroma contributes to tumor growth, angiogenesis, invasion, and therapeutic resistance in PDAC [31]. Thus, studies over the past two decades have revealed that the TME is an equally important hallmark of tumor behavior [7]. The TME is composed of different cellular populations, such as fibroblasts, immune and endothelial cells, among others. Fibroblasts in human tumors, also referred to as CAFs, play a key role in cancer development and progression in a variety of human tumors [8-10], including thyroid cancer.

\section{THYROID CANCER-ASSOCIATED FIBROBLASTS}

\section{Recruitment and activation of fibroblasts in thyroid cancer}

Fibroblasts are the major cellular components of the TME. In healthy tissues, fibroblasts are quiescent, but in the TME, they are reprogrammed by the tumor to acquire tumor supporting phenotypes. Fibroblasts can be stimulated by a variety of oncogenic signals to acquire an activated state, also referred to as myofibroblasts or CAFs. Compared with quiescent fibroblasts, CAFs are distinguished by their morphology, their metabolic, synthetic, and secretory phenotypes, as well as their enhanced proliferative and migratory properties and ECM production [810,32]. In contrast to quiescent fibroblasts, CAFs are larger, with more cytoplasm branches. Several protein markers are used to detect CAFs, including of $\alpha$-smooth muscle actin ( $\alpha$-SMA), platelet-derived growth factor receptor (PDGFR)- $\alpha / \beta$, fibroblast activation protein (FAP), vimentin, and neuron glial antigen (NG2). However, most of these markers are not unique to CAFs [9], making their identification challenging in some situations.

\section{What is known about the origin of CAFs?}

Numerous studies suggest that CAFs represent a heterogeneous component of the stroma and originate from multiple types of cells. A large number of them derive from mesenchymal stem cells and host tissue fibroblasts (pre-existing resident fibroblasts), which proliferate and expand locally after stimulation caused by cancer-derived growth factors. In addition, oncogenic signals produced by tumor cells may recruit fibroblasts to the tumor site from a distant source [8,9].

\section{What is known about the origin of thyroid CAFs?}

CAFs have been found to be abnormally increased in thyroid carcinomas as compared to normal thyroid tissue. The majority of studies investigating CAFs in human thyroid cancer correlate the expression of CAF-related proteins with clinical and pathological features. In a study performed in a small sample group of patients with PTC, the presence of CAFs (defined by $\alpha-S M A$ staining) was associated with cervical lymph node metastasis [33]. In another study, the expression levels of different CAFrelated proteins in human PTC was studied by using tissue microarrays, finding differences according to histologic subtypes and the presence of $B R A F^{V 600 E}$ mutation. Interestingly, PTCs with the $B R A F^{V 600 E}$ mutation exhibited high expression of some CAF-related proteins, such as FAP- $\alpha$. This study also revealed that stromal positivity for PDGFR- $\beta$ was associated with shorter overall survival in PTC [34]. In a more recent study, the presence of CAFs was investigated using immunostaining in human thyroid cancer. Higher levels of $\alpha$-SMA-positive areas were found preferentially localized at the tumor invasive front, in PTC and ATC. However, these studies did not find a specific or clear association between CAFs and a particular type of thyroid carcinoma [35]. Conversely, a very rare or absent infiltration of CAFs was described by $\alpha$-SMA immunostaining in a study performed on patients with ATC [36]. Interestingly, a PTC murine model of oncogenic BRAF ${ }^{\mathrm{V} 600 \mathrm{E}}$ activation in thyroid follicular cells showed increased fibroblast infiltration in thyroid cancer compared to normal thyroid tissue, in agreement with most observations in human tissue [37]. A higher density of $\alpha-\mathrm{SMA}^{+}$fi- 
broblasts was also found in a mouse model of PTC where thyroid-specific expression of oncogenic BRAF is activated and Pten is lost (BRAF V600E $/$ Pten $^{-1-} /$ TPO-Cre) [38]. Thus, these studies demonstrate recruitment and infiltration of CAFs into the TME in different types of thyroid carcinomas.

Once CAFs are accumulated in the TME, evidence suggests that they may subsequently continue to be attracted and/or activated in response to paracrine factors released from the neighboring tumor cells. For example, platelet-derived growth factor (PDGF), interleukin-6 (IL-6), reactive oxygen species (ROS), and transforming growth factor- $\beta$ (TGF- $\beta$ ), among others, are key regulators of fibroblast recruitment and activation [8-11].

\section{How are thyroid CAFs generated?}

It has been established that interaction between cancer cells and fibroblasts can promote the CAF phenotype in diverse tumor types. Unfortunately, the biology of tumor-stroma crosstalk in human thyroid cancer is largely unexplored. In a study performed on patients with PTC, a significant correlation was found between higher TGF- $\beta 1$ in cancer cells and increased $\alpha$-SMA levels in surrounding fibroblasts [39]. To recapitulate the effects of tumor cell secretome on stromal cells, our laboratory utilized in vitro co-cultures of human thyroid cancer and human fibroblast cell lines. We found that thyroid cancer cellderived conditioned media $(\mathrm{CM})$ reprogramed the thyroid fibroblast phenotype (Fig. 1) [40]. As mentioned before, CAFs are more proliferative than quiescent fibroblasts. Indeed, the number of human fibroblasts was significantly increased by treatment with thyroid cancer cell-derived CM [40]. Reprogramming fibroblasts into CAFs also involves alterations in their phenotype. We also demonstrated that thyroid cancer cell-derived $\mathrm{CM}$ induced up-regulation of CAF markers such as PDGFR- $\beta, \alpha$-SMA, and vimentin in human thyroid fibroblasts [40]. It has been demonstrated that, when transformed, CAFs show important metabolic alterations. In fact, glucose transporter 1 (GLUT-1) was markedly increased in human thyroid fibroblasts when cultured with thyroid cancer cell-derived CM (Fig. 1) [40]. Interestingly, we found that in addition to IL-6 and ROS, thyroid cancer cells secreted high amounts of PDGF into the media. All these soluble factors could serve as causative molecules that activate fibroblasts [40]. In line with our findings, Jolly et al. [38] reported that CM collected from thyroid cancer cells generated from $B R A F^{V 600 E} /$ Pten $^{-1} /$ TPO-Cre mice significantly increased proliferation and migration of murine CAFs. Collectively, these data suggest that tumor-derived soluble factors can induce a CAF phenotype in thyroid carcinoma

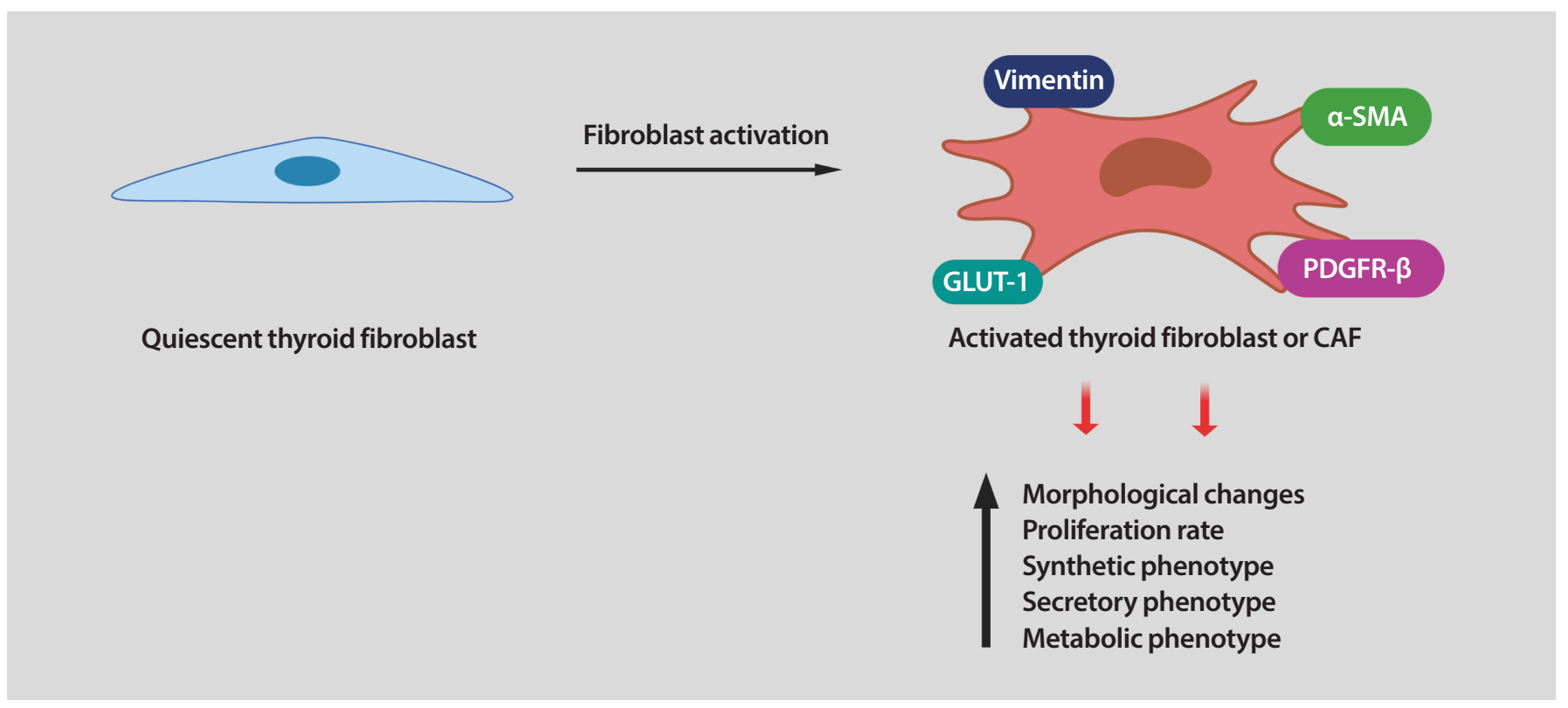

Fig. 1. Activation of thyroid fibroblasts. Cancer cells produce cytokines, chemokines, and growth factors to stimulate the transformation of quiescent or resting fibroblasts into activated cancer-associated fibroblasts (CAFs). Reprogramming of CAFs involves the increase in the levels of $\alpha$-smooth muscle actin ( $\alpha$-SMA), vimentin, and platelet-derived growth factor receptor $\beta$ (PDGFR- $\beta$ ), among other possible CAF markers. In addition, activated fibroblasts increase their proliferative properties and reprogram their secretory and metabolic phenotypes. Graphic created using BioRender. GLUT-1, glucose transporter 1. 
(Fig. 1). Nonetheless, many key questions still remain unsolved. For example, in addition to the paracrine signals generated by tumor cells, can cues released by other cells in the TME also contribute to fibroblast activation? Further studies are required to explore other TME-derived factors in thyroid cancer cells as well as the pathways involved in phenotypic reprogramming of human thyroid fibroblasts.

\section{Crosstalk between tumor cells and CAFs: tumor- stimulatory effects of CAFs in thyroid cancer}

As tumors grow, CAF cell numbers increase and accumulate. Once CAFs are reprogrammed, they influence most of the hallmark capabilities of cancer cells, playing key roles in the process of cancer development and progression through a variety of mechanisms. CAFs can exert their tumorigenic functions directly by cell-cell contact and indirectly by utilizing secreted factors. The array of functions and the mechanisms by which CAFs promote tumor progression are outside the scope of this review, but have been discussed in detail elsewhere [8-11,32]. However, it is important to point it out that CAFs are a considerable source of multiple secreted factors. So, we will focus on the CAF secretome and how it influences cancer cell behavior to support malignant thyroid tumor growth.

\section{What are the functions of CAFs in thyroid carcinogenesis?}

Only a few studies have provided direct evidence of the pro-tumorigenic role of CAFs in thyroid cancer. Using an in vivo subcutaneous model, Saitoh et al. [41] demonstrated the crucial role of the interaction between thyroid tumor cells and skin fibroblasts to promote the growth of tumors, in rats. According to the model, co-injection of tumorigenic Fisher rat thyroid follicular cells (FRTL-Tc) mixed with skin fibroblasts resulted in subcutaneous tumors larger than those derived from FRTL-Tc cells alone. Interestingly, the authors reported that fibroblasts enhance the growth of thyroid carcinoma by secreting soluble factors, although they did not identify any of them [41]. Another important contribution in understanding the functional effects of soluble factors released by CAFs on thyroid tumor progression has recently been provided by our laboratory. By using in vitro co-cultures, we have found that thyroid cancer cells treated with CAFs CM showed increased proliferation and invasion. In addition, we determined that soluble factors secreted by CAFs were able to activate epithelial-to-mesenchymal transition (EMT) in thyroid cancer cells. Classical mitogens for epithelial cancer cells, such as IL-6, were highly expressed and secreted by CAFs. We also found that ROS are abundantly produced by pro-tumor- igenic CAFs and secreted into the media [40]. Secretion of IL-6 and ROS by CAFs, among other soluble factors, are believed to promote thyroid tumor aggressiveness.

Another distinctive feature of CAFs is their ability to produce and deposit ECM. Alterations in the composition and crosslinking of the ECM change the stiffness of the tissue, thereby critically regulating tumorigenesis. The main constituents of the ECM are collagens [9]. Twenty-eight different collagens have been identified [42], and their assembly is highly regulated by the activity of enzymes, such as lysyl oxidase (LOX) [43]. Interestingly, LOX, an enzyme responsible for collagen crosslinking, was found to be highly expressed in aggressive thyroid carcinomas as compared to DTCs and normal thyroid tissues $[35,44]$. In addition, LOX inhibition significantly reduced the migration and invasion of ATC cell lines [44]. A recent study reported that BRAF-mutant tumors with high LOX expression were associated with more aggressive disease in thyroid cancer [45]. Consistent with these observations, in thyroid tumors from $B R A F^{V 600 E}$ murine models LOX expression levels were significantly higher than in normal tissue [38]. However, our current understanding of LOX and how it interacts with CAFs in thyroid cancer is still primitive, and more research is needed in order to design potential novel anti-tumor strategies based on LOX targeting for thyroid cancer.

Together, thyroid CAFs participate in a dynamic and interactive interplay with thyroid neoplastic cells. By secreting soluble factors (ROS, PDGF, and IL-6) thyroid tumor cells activate quiescent fibroblasts into CAFs. After activation, CAFs initiate the secretion of soluble factors and increase the ECM production, interacting with thyroid cells to foster malignant thyroid tumor growth by promoting cell proliferation, invasion, and EMT (Fig. 2).

\section{Implications of targeting thyroid CAFs: therapeutic perspectives}

Thyroid carcinoma, the most common form of endocrine malignancy, has an increasing incidence worldwide. While DTCs usually have a good prognosis, ATC is one of the most lethal solid tumors known. Conventional treatments of advanced thyroid cancer remain very challenging, and additional research is needed to find new treatment strategies to improve patient outcome.

Accumulating evidence indicates that CAFs exert pro-tumorigenic functions, making the different mechanisms that CAFs use to stimulate cancer cells exceptional therapeutic targets for cancer treatment [46]. Most strategies to manipulate CAFs to treat cancer focus on interfering with activation of CAFs or in- 


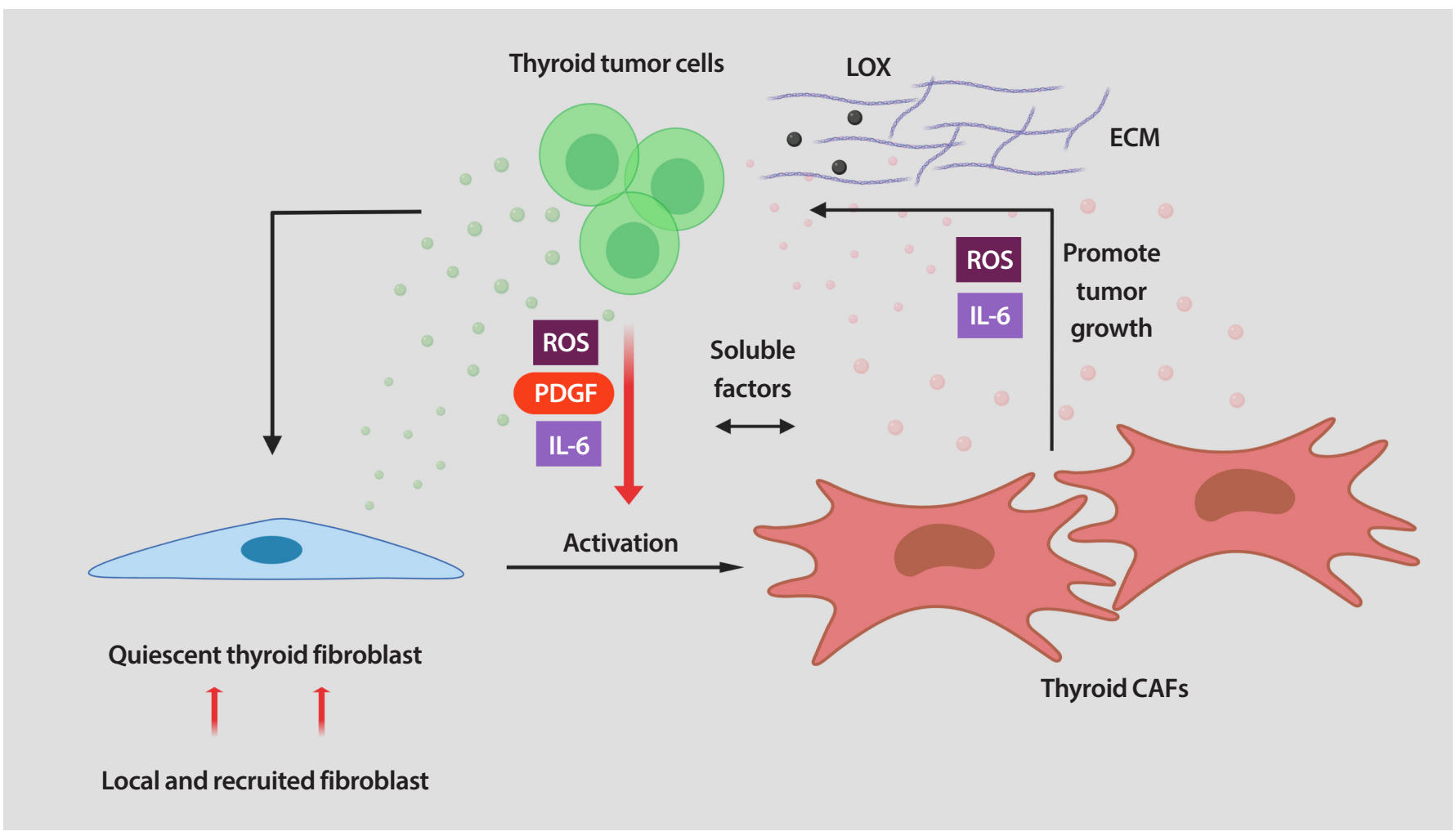

Fig. 2. A schematic model describing the dynamic interplay between cancer-associated fibroblasts (CAFs) and thyroid tumor cells. Thyroid tumor progression needs a positive feedback between CAFs and cancer cells. Cancer cells induce and maintain the fibroblasts' activated phenotype which, in turn, produces a series of growth factors and cytokines that sustain thyroid cancer progression by promoting cell proliferation and cell invasion. Graphic created using BioRender. IL-6, interleukin-6; ROS, reactive oxygen species; PDGF, platelet-derived growth factor; LOX, lysyl oxidase; ECM, extracellular matrix.

hibiting CAF functions. Several combinatorial strategies targeting TGF- $\beta$, which regulates the activation of CAFs, are currently being tested in clinical trials. For example, galunisertib, a TGF- $\beta$ receptor inhibitor, resulted in improved survival of patients with pancreatic cancer [47]. Inhibiting oncogenic signals secreted by CAFs by inhibitors or antibodies represents another attractive therapeutic strategy. Inhibitors of IL-6 are currently in use in different clinical trials and may be useful in combination therapies to treat cancer [48]. These observations highlight the potential of a wide range of therapeutic strategies targeting CAFs.

\section{CONCLUSIONS}

The role of the TME, particularly CAFs, in the development of thyroid cancer has long been underestimated. There is now more evidence clearly suggesting that interactions between CAFs and cancer cells are involved in thyroid cancer progression. Indeed, some of the mechanisms that these cells of the
TME use to promote thyroid tumor progression have recently been identified. These findings have made CAFs a promising new therapeutic target in thyroid cancer. Even so, numerous unanswered questions remain regarding CAFs in the thyroid cancer field. We believe that better understanding of thyroid CAFs' activation and function, as well the relationship between CAFs and thyroid cancer cells, is needed to design improved anti-cancer therapies in the future. These novel strategies can then be combined to enhance thyroid cancer patient outcome.

\section{CONFLICTS OF INTEREST}

No potential conflict of interest relevant to this article was reported.

\section{ACKNOWLEDGMENTS}

We regret any reference omissions due to length limitation. We thank Dr. Ramiro Iglesias-Bartolome of the National Cancer In- 
stitute for his critical reading of this manuscript.

The present research was supported by research grants from Agencia Nacional de Promoción Científica y Tecnológica (PICT 2015-0340 to Laura Fozzatti). Sheue-yann Cheng was supported by the Intramural Research Program of Center for Cancer Research at the National Cancer Institute, NIH.

\section{ORCID}

Laura Fozzatti https://orcid.org/0000-0003-0572-1977

Sheue-yann Cheng https://orcid.org/0000-0003-3530-2386

\section{REFERENCES}

1. National Cancer Institute. SEER cancer stat facts: thyroid cancer [Internet]. Bethesda: NCI; 2020 [cited 2020 Oct 14]. Available from: https://seer.cancer.gov/statfacts/html/thyro. html.

2. Davies L, Welch HG. Current thyroid cancer trends in the United States. JAMA Otolaryngol Head Neck Surg 2014; 140:317-22.

3. Siegel RL, Miller KD, Jemal A. Cancer statistics, 2020. CA Cancer J Clin 2020;70:7-30.

4. Fagin JA, Wells SA Jr. Biologic and clinical perspectives on thyroid cancer. N Engl J Med 2016;375:1054-67.

5. Cabanillas ME, McFadden DG, Durante C. Thyroid cancer. Lancet 2016;388:2783-95.

6. Hanahan D, Weinberg RA. Hallmarks of cancer: the next generation. Cell 2011;144:646-74.

7. Hanahan D, Coussens LM. Accessories to the crime: functions of cells recruited to the tumor microenvironment. Cancer Cell 2012;21:309-22.

8. Cirri P, Chiarugi P. Cancer associated fibroblasts: the dark side of the coin. Am J Cancer Res 2011;1:482-97.

9. Ohlund D, Elyada E, Tuveson D. Fibroblast heterogeneity in the cancer wound. J Exp Med 2014;211:1503-23.

10. Kalluri R. The biology and function of fibroblasts in cancer. Nat Rev Cancer 2016;16:582-98.

11. Sahai E, Astsaturov I, Cukierman E, DeNardo DG, Egeblad M, Evans RM, et al. A framework for advancing our understanding of cancer-associated fibroblasts. Nat Rev Cancer 2020;20:174-86.

12. Virchow R. Die Cellularpathologie in lhrer Begruendung auf Physiologische und Pathologische Gewebelehre. Berlin: Hirschwald; 1858.

13. Kalluri R, Zeisberg M. Fibroblasts in cancer. Nat Rev Can- cer 2006;6:392-401.

14. Mueller MM, Fusenig NE. Friends or foes: bipolar effects of the tumour stroma in cancer. Nat Rev Cancer 2004;4:83949.

15. Desmouliere A, Redard M, Darby I, Gabbiani G. Apoptosis mediates the decrease in cellularity during the transition between granulation tissue and scar. Am J Pathol 1995; 146:5666.

16. Tomasek JJ, Gabbiani G, Hinz B, Chaponnier C, Brown RA. Myofibroblasts and mechano-regulation of connective tissue remodelling. Nat Rev Mol Cell Biol 2002;3:349-63.

17. Dvorak HF. Tumors: wounds that do not heal. Similarities between tumor stroma generation and wound healing. N Engl J Med 1986;315:1650-9.

18. Cancer Genome Atlas Research Network. Integrated genomic characterization of papillary thyroid carcinoma. Cell 2014;159:676-90.

19. Suzuki H, Willingham MC, Cheng SY. Mice with a mutation in the thyroid hormone receptor beta gene spontaneously develop thyroid carcinoma: a mouse model of thyroid carcinogenesis. Thyroid 2002;12:963-9.

20. Cheng SY, Leonard JL, Davis PJ. Molecular aspects of thyroid hormone actions. Endocr Rev 2010;31:139-70.

21. Landa I, Ibrahimpasic T, Boucai L, Sinha R, Knauf JA, Shah $\mathrm{RH}$, et al. Genomic and transcriptomic hallmarks of poorly differentiated and anaplastic thyroid cancers. J Clin Invest 2016;126:1052-66.

22. Molinaro E, Romei C, Biagini A, Sabini E, Agate L, Mazzeo $\mathrm{S}$, et al. Anaplastic thyroid carcinoma: from clinicopathology to genetics and advanced therapies. Nat Rev Endocrinol 2017;13:644-60.

23. Xu B, Fuchs T, Dogan S, Landa I, Katabi N, Fagin JA, et al. Dissecting anaplastic thyroid carcinoma: a comprehensive clinical, histologic, immunophenotypic, and molecular study of 360 cases. Thyroid 2020;30:1505-17.

24. Ceolin L, Duval MADS, Benini AF, Ferreira CV, Maia AL. Medullary thyroid carcinoma beyond surgery: advances, challenges, and perspectives. Endocr Relat Cancer 2019; 26:R499-518.

25. Nikiforov YE, Nikiforova MN. Molecular genetics and diagnosis of thyroid cancer. Nat Rev Endocrinol 2011;7:56980.

26. Xing M. Molecular pathogenesis and mechanisms of thyroid cancer. Nat Rev Cancer 2013;13:184-99.

27. Pozdeyev N, Gay LM, Sokol ES, Hartmaier R, Deaver KE, Davis S, et al. Genetic analysis of 779 advanced differenti- 
ated and anaplastic thyroid cancers. Clin Cancer Res 2018; 24:3059-68.

28. Haugen BR, Alexander EK, Bible KC, Doherty GM, Mandel SJ, Nikiforov YE, et al. 2015 American Thyroid Association management guidelines for adult patients with thyroid nodules and differentiated thyroid cancer: the American Thyroid Association guidelines task force on thyroid nodules and differentiated thyroid cancer. Thyroid 2016;26:1133.

29. Hanahan D, Weinberg RA. The hallmarks of cancer. Cell 2000;100:57-70.

30. Quail DF, Joyce JA. Microenvironmental regulation of tumor progression and metastasis. Nat Med 2013;19:1423-37.

31. Schnittert J, Bansal R, Prakash J. Targeting pancreatic stellate cells in cancer. Trends Cancer 2019;5:128-42.

32. LeBleu VS, Kalluri R. A peek into cancer-associated fibroblasts: origins, functions and translational impact. Dis Model Mech 2018;11:dmm029447.

33. Cho JG, Byeon HK, Oh KH, Baek SK, Kwon SY, Jung KY, et al. Clinicopathological significance of cancer-associated fibroblasts in papillary thyroid carcinoma: a predictive marker of cervical lymph node metastasis. Eur Arch Otorhinolaryngol 2018;275:2355-61.

34. Sun WY, Jung WH, Koo JS. Expression of cancer-associated fibroblast-related proteins in thyroid papillary carcinoma. Tumour Biol 2016;37:8197-207.

35. Minna E, Brich S, Todoerti K, Pilotti S, Collini P, Bonaldi E, et al. Cancer associated fibroblasts and senescent thyroid cells in the invasive front of thyroid carcinoma. Cancers (Basel) 2020;12:112.

36. Caillou B, Talbot M, Weyemi U, Pioche-Durieu C, Al Ghuzlan A, Bidart JM, et al. Tumor-associated macrophages (TAMs) form an interconnected cellular supportive network in anaplastic thyroid carcinoma. PLoS One 2011;6:e22567.

37. Ryder M, Gild M, Hohl TM, Pamer E, Knauf J, Ghossein R, et al. Genetic and pharmacological targeting of CSF-1/CSF$1 \mathrm{R}$ inhibits tumor-associated macrophages and impairs BRAF-induced thyroid cancer progression. PLoS One 2013; 8:e54302.

38. Jolly LA, Novitskiy S, Owens P, Massoll N, Cheng N, Fang
W, et al. Fibroblast-mediated collagen remodeling within the tumor microenvironment facilitates progression of thyroid cancers driven by BrafV600E and Pten loss. Cancer Res 2016;76:1804-13.

39. Zhang J, Wang Y, Li D, Jing S. Notch and TGF- $\beta /$ Smad3 pathways are involved in the interaction between cancer cells and cancer-associated fibroblasts in papillary thyroid carcinoma. Tumour Biol 2014;35:379-85.

40. Fozzatti L, Alamino VA, Park S, Giusiano L, Volpini X, Zhao L, et al. Interplay of fibroblasts with anaplastic tumor cells promotes follicular thyroid cancer progression. Sci Rep 2019;9:8028.

41. Saitoh O, Mitsutake N, Nakayama T, Nagayama Y. Fibroblast-mediated in vivo and in vitro growth promotion of tumorigenic rat thyroid carcinoma cells but not normal Fisher rat thyroid follicular cells. Thyroid 2009;19:735-42.

42. Ricard-Blum S. The collagen family. Cold Spring Harb Perspect Biol 2011;3:a004978.

43. Tenti P, Vannucci L. Lysyl oxidases: linking structures and immunity in the tumor microenvironment. Cancer Immunol Immunother 2020;69:223-35.

44. Boufraqech M, Nilubol N, Zhang L, Gara SK, Sadowski SM, Mehta A, et al. miR30a inhibits LOX expression and anaplastic thyroid cancer progression. Cancer Res 2015;75: 367-77.

45. Boufraqech M, Patel D, Nilubol N, Powers A, King T, Shell $\mathrm{J}$, et al. Lysyl oxidase is a key player in BRAF/MAPK pathway-driven thyroid cancer aggressiveness. Thyroid 2019;29: 79-92.

46. Chen X, Song E. Turning foes to friends: targeting cancerassociated fibroblasts. Nat Rev Drug Discov 2019;18:99115 .

47. Melisi D, Garcia-Carbonero R, Macarulla T, Pezet D, Deplanque G, Fuchs M, et al. TGF $\beta$ receptor inhibitor galunisertib is linked to inflammation- and remodeling-related proteins in patients with pancreatic cancer. Cancer Chemother Pharmacol 2019;83:975-91.

48. Johnson DE, O'Keefe RA, Grandis JR. Targeting the IL-6/ JAK/STAT3 signalling axis in cancer. Nat Rev Clin Oncol 2018;15:234-48. 